\title{
Pratiques de branding en contexte universitaire. Une approche processuelle de la marque
}

Branding Practices in a University Context: A Processual Approach of the Brand

\section{Consuelo Vásquez, Benoit Cordelier et Viviane Sergi}

\section{(2) OpenEdition}

Journals

Édition électronique

URL : http://journals.openedition.org/edc/5143

DOI : $10.4000 /$ edc. 5143

ISSN : 2101-0366

Éditeur

Université de Lille

Édition imprimée

Date de publication : 1 juin 2013

Pagination : $93-110$

ISBN : 978-2-917562-09-3

ISSN : $1270-6841$

Référence électronique

Consuelo Vásquez, Benoit Cordelier et Viviane Sergi, « Pratiques de branding en contexte universitaire. Une approche processuelle de la marque », Études de communication [En ligne], 40 | 2013, mis en ligne le 01 juin 2013, consulté le 10 décembre 2020. URL : http://journals.openedition.org/edc/5143; DOI : https://doi.org/10.4000/edc.5143

Ce document a été généré automatiquement le 10 décembre 2020.

(c) Tous droits réservés 


\title{
Pratiques de branding en contexte universitaire. Une approche processuelle de la marque
}

\author{
Branding Practices in a University Context: A Processual Approach of the Brand
}

\author{
Consuelo Vásquez, Benoit Cordelier et Viviane Sergi
}

1 Le passage vers une société collaborative (Papilloud, 2007) transforme les modalités de communication des organisations. Ce qui relevait d'une pratique professionnelle délimitée et attribuée à des professionnels clairement identifiés se distribue aujourd'hui dans les pratiques de communication des acteurs de l'organisation. C'est le cas par exemple de la création et de la gestion d'une image ou marque de l'organisation. Les actions relevant d'une forme de ce qui, dans le milieu du marketing, s'appelle branding n'est plus l'apanage des professionnels. Ce constat favorise de nouvelles perspectives dans la manière d'analyser les pratiques du marketing et du branding. Celles-ci sont particulièrement influencées par les récents rééquilibrages dans le processus organisationnel qui aboutit à la production de la marque. Dans cet article, nous souhaitons étudier la production de pratiques et d'artefacts contribuant au développement d'une image de marque. Nous nous intéressons plus particulièrement à la manière dont ces assemblages reconfigurent l'organisation. En ce sens, nous postulons que le branding en tant que processus organisationnel va au-delà des pratiques et stratégies de marketing mais devient une forme particulière d'organisation, un mode d'ordonnancement basé sur la relation entre identité et image. Nous nous inspirons à cet effet de récentes études de théories sociologiques de la marque qui suggèrent que celle-ci est une interface complexe qui organise les relations entre les producteurs et les consommateurs (Arvidsson 2006, 2008 ; Lury, 2004 ; Moor, 2007). Nous nous concentrons sur la dimension organisante de la marque et montrons comment elle devient « une plateforme pour la modélisation des activités, un mode d'organisation des activités dans le temps et l'espace » (Lury, $2004: 1$, notre traduction).

2 Pour étudier la marque comme un mode d'organisation (organizing), nous proposons une approche verticale-ascendante (bottom-up) qui consiste à expliquer l'ordonnancement 
social à partir d'interactions au niveau micro. Tarde (2000 [1899]), un des premiers sociologues à avoir adopté une telle perspective (Cooren et Fairhurst, 2009), nous invite en effet à rendre compte des détails de la vie sociale puisque dans le monde des faits, l'origine de l'ordre se trouve dans la compilation de micro événements. Dans cette perspective, le branding est conçu comme un processus interactionnel de représentations à partir de pratiques spécifiques de production, de consommation et de distribution d'éléments de marque. Nous envisageons ainsi l'étude de la marque, au-delà des habituelles sphères commerciale, économique ou encore juridique, à travers l'analyse de pratiques communicationnelles quotidiennes qui forment le processus du branding.

À partir de cette approche de la marque, nous nous sommes attachés à une étude de cas de travail de branding en contexte universitaire. Ce terrain est intéressant en raison des récents efforts des universités pour accroitre leur visibilité à travers des activités de promotion et de positionnement en réponse à une marchandisation de plus en plus importante de ce secteur (Czarniawska et Genell, 2002). Nous nous intéressons plus particulièrement aux efforts de branding portés par le corps professoral d'un département d'une université nord-américaine. Nous montrons comment par un processus continu de négociations, les professeurs questionnent leur identité et l'image qu'ils veulent produire à travers, dans ce cas, leur site internet.

4 Notre appartenance au monde universitaire nous donne une compréhension fine des préoccupations et questionnements auxquels sont exposées les personnes de notre terrain. Cela est renforcé par le fait qu'au moment de la recherche, nous relevons d'institutions différentes aux enjeux diversifiés dont le traitement a enrichi nos points de vue et nous a permis d'établir un dialogue avec des personnes qui étaient, somme toute, nos collègues. Mais cela n'a pas été sans nous poser quelques difficultés car si nous sommes acceptés comme des membres, au sens de Garfinkel (1967), de la grande institution universitaire, nous pouvons également être perçus comme des personnes extérieures. En reprenant les catégories d'Adler et Adler (1987), nous pouvons dire que nous sommes engagés dans une observation participante périphérique.

5 L'intérêt de cet article réside dans sa contribution à la fois aux récents développements de la recherche en branding des universités et à l'établissement d'un modèle conceptuel de la marque en tant que processus interactionnel basé sur des pratiques communicationnelles. Dans la perspective de ce double apport, nous nous proposons de présenter le cadre théorique qui nous sert à développer notre approche du branding en tant que mode d'existence de l'organisation(I). Nous présentons ensuite notre méthodologie ainsi que le cas qui sert à notre analyse (II). Nous concluons sur l'intérêt de cette approche pour l'étude communicationnelle des organisations (III).

\section{Du branding en tant que mode d'existence}

\section{Universités et branding : pour une approche bottom-up}

$6 \quad$ L'intérêt croissant pour le branding des universités s'explique par les changements dans les modèles de fonctionnement des institutions universitaires qui tendent à devenir des industries à part entière (Gumport, 2000). Celui-ci est d'autant plus crucial que, d'un côté, il fait le lien entre identité et image et, que de l'autre, il est un facteur d'attraction et de fidélisation des étudiants comme des enseignants et des donateurs. Les universités 
doivent alors à la fois s'interroger sur les caractères qui les définissent ainsi que sur la perception qu'en ont leurs publics (Stensaker, 2007).

Pourtant, le branding des universités est encore un domaine peu exploré (Chapleo, 2010; Wærass et Sobakk, 2009). Le processus de production de la marque (la manière dont sont prises les décisions et les pratiques qui en découlent) l'est encore moins (Ali-Choudhury et al., 2009 ; Hemsley-Brown et Goonawardana, 2007). De plus, comme le précise Stensaker, le défi du branding dans les universités est « d'atteindre un équilibre entre la nécessité de s'ajuster à un monde en évolution tout en maintenant leurs identités et les caractéristiques inhérentes à l'éducation supérieure » (2007: 15, notre traduction). En raison du faible nombre d'études empiriques sur les pratiques de branding universitaire de telles conclusions restent vagues et laissent beaucoup de questions sans réponses. Nous pouvons ainsi nous interroger sur ces caractéristiques inhérentes ou sur ce que sont ces identités organisationnelles des universités, sur la manière dont elles prennent forme, dont elles sont mobilisées, sur la manière d'atteindre cet équilibre. Dans cette perspective, nous proposons de considérer que le processus de branding se déroule également sur des périodes qui ne sont pas forcément identifiées comme étant dédiées à la création de la marque. Il adopte donc de nombreuses modalités et a un caractère fondamentalement hybride qui n'exclut pas pour autant les activités de planification ou toute autre activité formelle relevant, par exemple, de la gestion de la marque. Nous souhaitons plutôt montrer comment ces actions se déroulent dans des interactions plus quotidiennes et participent d'un mode d'existence et de développement de l'organisation.

\section{Le branding comme organisation de re-présentations}

Sur le plan théorique, le concept de pratiques de représentation combine des apports de deux approches épistémologiquement proches : la perspective centrée sur les pratiques ( practice-based studies) et l'approche de la communication constitutive des organisations (CCO). L'étude des pratiques partagent un point d'intérêt central : l'analyse de ce qui est fait, de ce qui est accompli au quotidien par des individus ou des groupes qui, à terme, produisent des phénomènes macros (tels que la culture, la structure, les normes, etc.). Nous ancrons toutefois notre recherche plutôt dans le courant de la CCO (pour une revue récente de la littérature cf. Cooren et al., 2011) car nous mettons l'accent sur le rôle constitutif fondamental de la communication dans les phénomènes organisationnels tout en favorisant une approche verticale ascendante (bottom-up) du branding afin d'étudier les micro-pratiques qui construisent les liens entre l'identité et l'image d'une organisation. Dans cette perspective, nous mettons de plus en avant un point de vue performatif et interactionniste des organisations (Fairhurst et Putnam, 2004). Nous concevons les organisations comme le produit de pratiques sociales (Giddens, 1987), de textes (Taylor et al., 1996) et leurs dérivés, propriétés de la communication et de l'action (Putnam et Nicotera, 2009). Le branding est alors étudié comme un processus interactionnel et communicationnel de re-présentations ${ }^{1}$ où des pratiques spécifiques de production, de consommation et de distribution des marques entre des agents ou acteurs organisationnels puis des consommateurs. Malgré l'analogie forte avec le système marchand, le concept de consommateur doit ici être également compris comme celui d'un individu non pas tant qui achète la marque mais qui en fait usage (acte de consommation) au point éventuellement de la transformer. Par conséquent, que ce soit dans sa production comme dans sa consommation, nous nous éloignons de l'approche 
commerciale et économique plus habituelle de la marque pour tendre vers l'étude de pratiques de communication du faire la marque. Nous nous concentrons alors sur le processus de production de marque (branding) centré sur des pratiques quotidiennes de communication que nous appelons pratiques de re-présentation.

9 En résumé, nous envisageons le branding comme un ensemble de pratiques, un travail continu qui se déroule dans des instances préoccupées par des questions d'identités aussi bien organisationnelles qu'individuelles. L'identité de l'organisation n'est plus alors un état, une propriété, mais une production dynamique réalisée dans les interactions et doit être performée puis incarnée, matérialisée dans des micro-actions situées qui créent cette re-présentation. Ainsi, nous considérons que la vision d'une marque ou identité unique et homogène qui s'impose aux acteurs organisationnels par sa cohérence et sa consistance est une utopie. En suivant le courant de la $\mathrm{CCO}$, nous proposons d'explorer la multiplicité et l'hétérogénéité des re-présentations qui se confrontent, s'assemblent ou éclatent dans la co-construction de la marque. Cette confrontation, mode de transaction, est essentielle pour comprendre l'être et le devenir de l'organisation entre identités, images et branding.

\section{Éléments ethnographiques d'une production de site internet}

\section{Méthodologie}

10 Cet article s'appuie sur une recherche en-cours portant sur le branding en action dans une Université Canadienne (UC), et plus particulièrement une unité départementale (UD), où nous menons une ethnographie organisationnelle (Schwartzman, 1993 ; Boden, 1994). Si cette recherche étudie différentes facettes des travaux et pratiques de branding, nous nous concentrons ici sur la mise en place d'un site internet départemental.

11 La collecte de données ethnographiques démarre en janvier 2010. L'un des auteurs note des observations, compile des photographies de l'environnement physique de l'organisation afin de réunir une première collection de re-présentations, aussi bien de l'université que du département. Cette première étape se déroule jusqu'au printemps et permet, à travers la constitution d'une étude de cas, de nous familiariser avec les particularités de l'UC, son contexte et son histoire, d'en comprendre la culture et les modes de fonctionnement. Plus tard, l'opportunité d'observer à partir de l'automne suivant la mise en place d'un nouveau site internet au sein d'un département de l'UC apparaît alors prometteuse pour étudier plus finement des micro-pratiques de branding.

Dans le cadre de ce volet de la recherche, afin de mettre leurs actions en contexte, des entrevues semi-dirigées sont réalisées avec plusieurs membres de l'UD ainsi qu'avec la responsable des communications marketing de l'université. En parallèle, nous avons recueilli de nombreux documents administratifs et compilé plus de dix pages de courriels entre membres de l'UD au sujet de ses orientations stratégiques et du changement de site.

Enfin, nous avons également pris des notes lors de réunions départementales et plus particulièrement lors de celle pendant laquelle le nouveau site est présenté et discuté. Cette dernière réunion est essentielle car ce nouveau site matérialise et rend présent le Département en faisant un sujet sensible pour tous ses membres. Les réunions rendent non seulement l'organisation visible (Schwartzman, 1989 ; Boden, 1994), mais elles nous ont permis d'étudier de plus près les interactions menant aux pratiques de branding, de 
mettre en évidence les questions d'identités et de re-présentations ainsi que la manière dont elles sont mobilisées, reliées et assemblées lors des échanges autour du site internet.

\section{Étude cas : un nouveau site web départemental}

14 En avril 2010, dans le cadre d'un projet d'actualisation du site internet départemental, le directeur de l'UD rencontre les services audiovisuels de l'université afin d'explorer les différentes possibilités structurelles pour le développement d'un nouveau site. Il s'est notamment concentré sur la page d'accueil et la définition des courants de recherche qui caractérisent les professeurs de l'UD, auxquels il demande une participation active. L'un des objectifs est de donner à ces derniers une place centrale par la réalisation d'une mosaïque de leurs photographies afin de montrer que, selon les propos du directeur, «le département, c'est nous ». À ces fins, un courriel est envoyé aux professeurs autour de la semaine de relâche du printemps en leur demandant de prendre rendez-vous avec le photographe de l'université; il demande également un bref curriculum vitae avec une structure suggérée de façon à ce que le personnel administratif complète leurs pages. Le directeur demande dans un autre courriel à chaque professeur d'identifier les principaux axes de recherche et d'en sélectionner tout au plus deux auxquels il souhaite être associé. Par la suite, une proposition d'axes de recherche communs est faite à travers un nouveau courriel collectif. Ces courriels étaient suivis de messages privés ou de discussions entre le directeur et quelques-uns des professeurs qui menèrent à une redéfinition partielle des axes de recherche. Il est également intéressant de noter qu'au mois d'août les discussions furent enrichies par la présentation d'une version temporaire du site Web visible des seuls professeurs. Les commentaires pendant ce mois portèrent aussi bien sur la mosaïque que sur les courants de recherche.

Pour exposer les pratiques communicationnelles, objet de cet article, nous allons maintenant nous focaliser sur deux éléments communicationnels spécifiques : un courriel et une discussion dans une assemblée départementale.

\section{Un exemple de courriel}

Le courriel suivant (fig. 1) illustre plus particulièrement l'articulation entre les pratiques communicationnelles employées par le Directeur de l'UD afin d'établir les conditions de la discussion collective et de la prise de décision dans le processus de branding plus particulièrement concernant le site web. Le sujet de discussion, porte sur la mise en évidence des éléments à prendre en considération, des acteurs impliqués et des activités à réaliser. Dans l'analyse ci-dessous, nous faisons émerger les concepts des pratiques identifiées et nous les définissons ultérieurement.

Le Directeur établit le contexte de son message (« As I mentioned in a previous mail ») et définit l'idée qui doit diriger le processus de branding («the Department is first and foremost its professors »). Cette idée du « Département, c'est nous » est rappelée par le Directeur dans chacune de ses interventions (courriels, discussions, entretiens, réunions) sur le sujet. Le cadrage se fait sur la nécessité de rendre compte de l'importance des personnes dans l'identification de l'UD, afin de ne pas le concevoir exclusivement comme une unité administrative, une structure déshumanisée. Toutefois, la portée de ce nous change selon les interlocuteurs et le contexte. Il peut inclure ou pas, outre les professeurs, les chargés de cours et le personnel administratif. Si la délimitation ainsi faite 
laisse place à une évolution dans la matérialisation de ce nous, rapidement le ton affirmatif amène à détacher l'idée d'une mosaïque de visages et une liste de domaines d'expertise. Un travail de resserrement et de stabilisation des idées (ou couplage comme nous le définissons plus tard) est également fait. Les actions à entreprendre en découlent : photographie à prendre ("That's why we ask you to take pictures of you »), CV à envoyer (" the CV that [the secretary] asked you ») et identification des domaines d'expertise (" Also, could I ask you to validate the following breakdown of your respective expertise? »). Et le Directeur fait preuve de sa subtile connaissance de l'univers de ses collègues qui, bien qu'ils tendent en effet à vouloir commenter, critiquer tous les choix qui sont fait au nom du collectif, manquent de temps pour mener à bien toutes les actions qui en découlent et ont besoin de soutien ("This is to make your life easier ", "a few rules to follow») sur les aspects plus concrets de cette transformation. Il obtient ainsi l'engagement de ses collègues à travers un jeu de pratiques communicationnelles. Comme nous venons de le voir, celles-ci fonctionnent individuellement comme en combinaisons. Nous pourrions d'ailleurs encore en prolonger l'analyse sur ce simple courriel mais il nous semble également important d'exposer un autre contexte communicationnel où elles sont à l'œuvre : une réunion départementale.

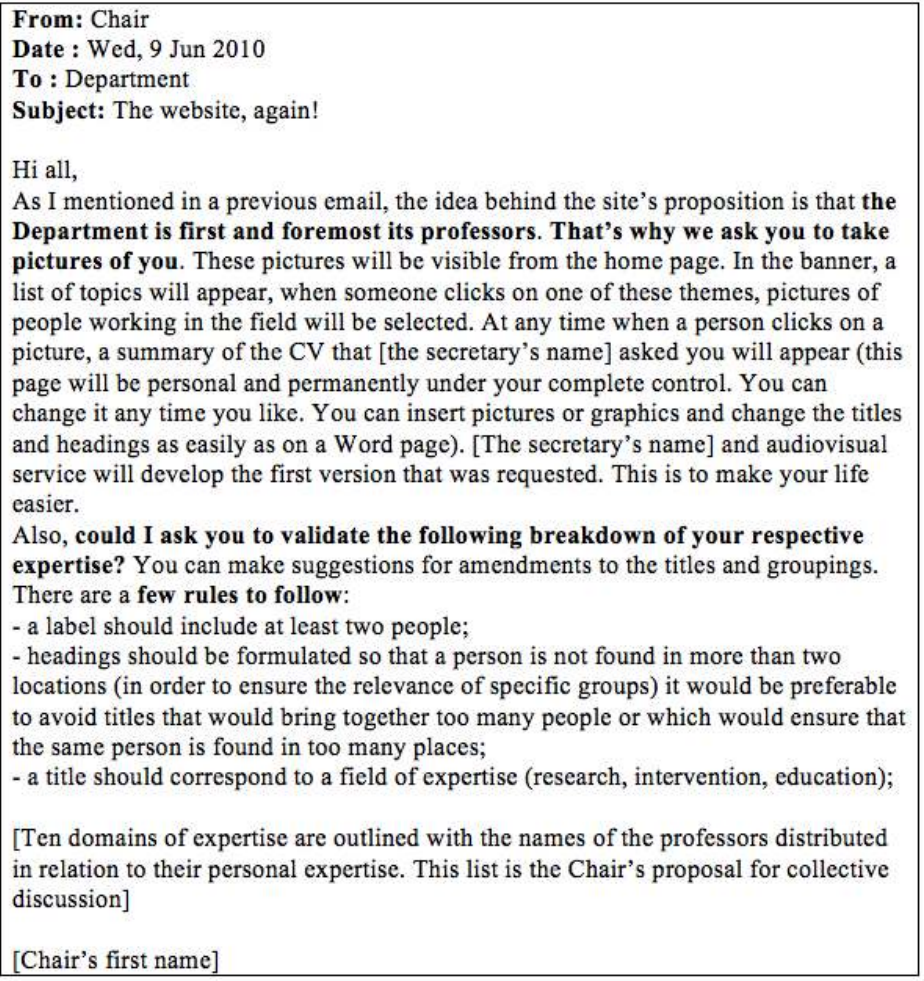

Figure 1 : Courriel du directeur de département

\section{L'assemblée départementale}

La présentation officielle du nouveau site web est faite lors de la première réunion départementale de 2010-2011. Cette rencontre d'une journée est l'occasion de développer des sujets clés et de prendre des décisions importantes au sujet du plan stratégique et des offres de postes pour les prochaines années. Le sujet du site internet est abordé après le déjeuner et porte essentiellement sur des points techniques concernant les options de modification des pages personnelles des professeurs. 
n'apparaissaient pas sur le site Internet, ce à quoi le directeur répond qu'il a dû faire des choix en essayant de respecter les intentions des collègues et l'esprit de l'exercice ( couplage). Les discussions tournent autour des choix esthétiques du site. La représentation des chargés de cours et des professeurs associés (délimitation) est repoussée pour laisser du temps à la présentation des caractéristiques techniques en raison de la présence d'un technicien du service audiovisuel (cadrage). Pendant sa présentation, les explications sont interrompues par les questions des professeurs qui font des liens entre des points techniques et des problématiques liées à leurs représentations. Ceux-ci tentent de comprendre et d'explorer les possibilités qu'ils ont pour proposer des conceptions alternatives du site et plus particulièrement de la page de la mosaïque (cadrage). Celle-ci provoque en effet des critiques négatives. Toutefois, la discussion fut recentrée exclusivement sur les questions techniques qui relèvent des compétences du technicien ( cadrage). Les débats sont également contraints par la nécessité de prendre d'autres décisions sur des sujets très importants (comme l'offre de postes) avec des échéances serrées (cadrage).

À la fin de la réunion, alors que des professeurs s'interrogent sur les suites concernant les débats autour du site internet, et que d'autres quittent la réunion, le directeur souligne que c'est un travail en cours, et il repousse la discussion pour une prochaine réunion tout en demandant et obtenant la permission de mettre en ligne le site en l'état (couplage). Un vote à main levée est rapidement organisé, la majorité accepte et la réunion se clôt ( couplage). Le jour suivant, le site web est mis en ligne et à date, la future réunion n'a pas encore eu lieu alors que plusieurs des problèmes soulevés n'ont pas été résolus.

\section{Une analyse communicationnelle de la pratique de branding}

\section{Disputes entre unification et fragmentation}

Rappelons d'abord que nous considérons le branding comme un processus articulant un ensemble de pratiques de communication, une forme de travail collectif continu où les questions d'identités individuelles et organisationnelles sont imbriquées avec des problématiques de représentations organisationnelles. Nous passons ainsi de l'idée de l'identité organisationnelle comme attribut à celle d'une production dynamique (Hatch et Schultz, 2002, 2008; Gioia, Schultz et Corley, 2000) qui doit être performée. Notre définition nous amène à étudier directement le processus du branding comme un ensemble de pratiques de mobilisation et d'élaboration de représentations, ces pratiques étant situées, hétérogènes et collectives.

Les discussions qui ont mené à la mise en place du nouveau site web du Département sont caractérisées par une dialogique de fragmentation/unification de l'identité collective. La notion d'identité collective doit ici être comprise comme un construit social qui s'élabore à travers des pratiques discursives situées (Ainsworth et Hardy, 2004 ; Grant et al., 2004 ; Brown, 2006; Kuhn, 2006) qui tiennent compte de multiples narrations (Cunliffe et al., 2004). L'objectif de départ du site est de mettre en évidence le caractère distinctif de l'identité de l'UD en présentant une image claire et unifiée du " qui ils sont » en tant que collectif. Il est intéressant de remarquer que bien que la question de l'identité du

Études de communication, 40 | 2013 
Département n'ait été que peu explicitement et ouvertement discutée, elle est présente en filigrane tout au long du processus de branding et n'a pas trouvé de véritable résolution pour de nombreux professeurs. Tout au long du processus, ces derniers essaient de créer une identité unifiée où chaque proposition est immédiatement suivie par une opposition, une forme de résistance à cette tentative de réduction de la diversité. Il est par conséquent peu surprenant que la proposition qui finit par émerger ne soit pas fortement consensuelle.

Les réactions peuvent être interprétées à deux niveaux. Tout d'abord, certaines d'entre elles sont directement en lien avec le nous qui est créé dans la nouvelle page web puisque les professeurs perçoivent un écart entre ce qu'ils considèrent être leur identité départementale et l'image projetée par le site. D'un autre côté, d'autres réactions peuvent être interprétées comme un écho au processus qui a mis le nous en évidence. Non sans une certaine ironie, l'exercice du site Web a révélé non seulement l'identité de l'UD, mais a surtout mis en évidence les difficultés pour unifier cette identité collective et par conséquent, l'hétérogénéité de ces nous.

\section{Pratiques de représentation}

Pour illustrer notre propos, nous venons d'exposer ici deux épisodes qui ont contribué au processus de branding de l'UD et qui nous permettent de mettre en évidence les pratiques communicationnelles à l'œuvre. En nous concentrant («zooming in », Nicolini, 2009) sur l'événement communicationnel spécifique, nous retraçons les progrès discursifs et matériels qui ont contribué à la conception du site. L'étude des actions concrètes de ce travail, nous a permis de conceptualiser trois pratiques communicationnelles : le cadrage, la délimitation et le couplage. Celles-ci ont donné la possibilité de découvrir le travail continu de mise en avant des problématiques identitaires sur le plan organisationnel et individuel. Cette démarche permet de rendre compte à la fois des efforts pour énacter la nouvelle marque et des effets sur ceux qui ont participé à son élaboration. Nous révélons ainsi la dynamique d'alignement des identités et des images produites. Ces pratiques permettent de décortiquer comment la marque agit et est énactée par des pratiques de cadrage, de délimitation et de couplage pour identifier les personnes et l'objet de la marque.

Le cadrage est ici définit comme une pratique communicationnelle par laquelle «les personnes créent des distinctions entre ce qui est à l'avant plan et à l'arrière plan dans une situation" (Brummans et al., 2008: 26, notre traduction ; cf. également Goffman, 1974 ; Bateson, 1955/1972; Dewulf et al., 2009, 2011). Le cadrage est donc inhérent à l'activité de sensemaking quotidienne des individus. Associées au processus de branding, les dynamiques communicationnelles du cadrage sont orientées par la marque. Cette dernière permet alors d'établir un cadre pour donner un sens à une situation, en rendre compte et permettre de choisir ce qui doit être fait.

La délimitation correspond davantage à un travail d'inclusion/exclusion à l'instar de la clôture communicationnelle. Pour Hernes, l'organisation « émerge dans l'établissement d'une distinction et persiste par l'établissement de frontières" (2004: 8, notre traduction). Nous définissons dès lors la délimitation comme une pratique communicationnelle à travers laquelle les individus tracent des frontières entre eux et ce qui doit être pris en considération dans le processus de branding. La délimitation dans le branding contribue à la nature organisante de la marque qui inclut/exclut, assemble/ démonte des activités et des acteurs dans le temps et l'espace. 

termes d'attributs. Elle oriente les analyses théoriques des pratiques communicationnelles et professionnelles vers des perspectives plus praxéologiques. Elle rappelle sur un plan managérial différentes dynamiques dont il faut jouer lors du développement d'une identité organisationnelle et de sa réification dans les vecteurs de marque.

31 En somme, nous proposons une approche du branding comme étant un processus articulé autour de pratiques communicationnelles telles que celles que nous avons identifié ici : cadrage, délimitation et couplage. Est-ce que cela peut toujours être considéré comme du branding? Nous le croyons puisqu'il soulève des questions autour de l'identité et il vise à établir une représentation (plus ou moins) cohérente, dans notre cas d'un département d'université. En nous concentrant sur la manière dont un nouveau site web a été développé à travers le temps, nous avons mis en évidence les réalités communicationnelles du branding et ses implications: une marque mise en action, un amalgame d'identités et d'images créées dans des interactions autour de multiples acteurs. Dans ce cas, d'important efforts ont été consacrés à aligner identité, image, branding (Hatch et Schultz, 2002, 2008) à travers des pratiques de représentation. Pourtant, de nombreuses dimensions caractérisées aussi bien par leurs singularités que par leur conflictualité étaient entremêlées dans ce processus: attentes et objectifs personnels comme collectifs, échéances réduites, contraintes techniques et politiques. 
Cela explique en partie pourquoi les images et les marques ne sont pas stables mais l'objet d'un processus continu. Elles sont le résultat de négociations constantes visant à mettre de l'ordre dans des systèmes de significations variées.

\section{BIBLIOGRAPHIE}

Adler P. et Adler P., (1987), Membership roles in field Research, Sage Publications, U.S.A., 96 p.

Ainsworth S. et Hardy C., (2004), Discourse and identities, in Grant D. et al. (éds.), Handbook of organizational discourse, London, Sage, pp. 153-173.

Ali-Choudhury R., Bennett R. et Savani S., (2008), University marketing chairs' views on the components of a university brand, in International Review on Public and Nonprofit Marketing, 6(1), pp. 11-33.

Alvesson M., (1990), Organizations : From substance to image, in Organization Studies, 11(3), pp. 373-394.

Ardvidsson A., (2008), The Ethical Economy of Customer Coproduction, in Journal of Macromarketing, December, 28(4), pp. 326-338.

Ardvidsson A., (2006), Brand : Meaning and value in media culture, London : Routledge, 176 p.

Boden D., (1994), The Business of Talk, Cambridge (U.K.), Polity Press, 288 p.

Brown A. D., (2006), A narrative approach to collective identities, in Journal of Management Studies, 43/4, pp. 731-753.

Chapleo C., (2010), What defines « successful » university brands ?, in International Journal of Public Sector Management, 23(2), pp. 169-183.

Cooren F. et Fairhurst G., (2009), Dislocation and stabilization : How to scale upfrom interactions to organization, in L. L. Putnam et A. M. Nicotera (éds.), Building theories of organization. The constitutive role of communication, (pp. 117-152), Mahwah, NJ, Lawrence Erlbaum.

Cunliffe A. L., Luhman J. T. et Boje D. M., (2004), Narrative temporality : Implications for organizational research, in Organization Studies, 25/2, pp. 261-286.

Czarniawska B. et Genell K., (2002), Gone shopping ? Universities on their way to the market, in Scandinavian Journal of Management, 18, pp. 455-474.

Dewulf A., Gray B., Putnam L. L., Lewicki R., Aarts N., Bouwen R. et Van Woerkum C., (2009), Disentangling approaches to framing in conflict and negotiation research : A meta-paradigmatic perspective, in Human Relations, 62(2), pp. 155-193.

Dewulf A., Gray B., Putnam L. L. et Bouwen R., (2011), An interactional approach to framing in conflict and negotiation, in Donohue W. A., Rogan R. G. et Kaufman S. (éds.), Framing matters : Perspectives on negotiation research and practice in communication, Peter Lang, New York, pp. 7-33.

Garfinkel H., (1967), Studies in Ethnomethodology, Englewood Cliffs, Prentice Hall, 368 p.

Giddens A., (1987), La constitution de la société, Paris, Presses universitaires de France, 474 p. 
Gioia D., Schultz M. et Corley K., (2000), Organizational identity, image, and adaptive instability, in Academy of Management Review, 25(1), pp. 63-81.

Goffman E., (1974), Frame analysis : An essay on the organization of experience, Northeastern University Press, Boston, MA, $586 \mathrm{p}$.

Golsorkhi D., Rouleau L., Seidl D. et Vaara E., (2010), Cambridge Handbook of Strategy-as-Practice, Cambridge, Cambridge University Press, 368 p.

Gumport P., (2000), Academic restructuring: Organizational change and institutional imperatives, in Higher Education, 39, pp. 67-91.

Grant D., Hardy C., Oswick C. et Putnam L., (2004), Introduction, Organizational discourse :

Exploring the field, in D. Grant et al. (éds.), Handbook of organizational discourse, London, Sage, pp. 1-36.

Hatch M. J. et Schultz M., (2002), The dynamics of organizational identity, in Human Relations, 55 (8), pp. 989-1018.

Hatch M. J. et Schultz M., (2008), Taking Brand Initiative : How Companies can Align their Strategy, Culture and Identity through Corporate Branding, San Francisco, Jossey-Bass, 288 p.

Hemsley-Brown J. et Goonawardana S., (2007), Brand harmonization in the international higher education market, in Journal of Business Research, 60(9), pp. 942-948.

Hernes T., (2004), Studying composite boundaries : a framework for analysis, in Human Relations, 57 (1), pp. 9-29.

Kuhn T., (2006), A « demented work ethic » and a « lifestyle firm » : "Discourse, identity, and workplace time commitments », in Organization Studies, 27/9, pp. 1339-1358.

Latour B., (2013), "What's the story? ", Organizing as a mode of existence, in Robichaud D. et Cooren F. (éds.), Organization and organizing : Materiality, Agency and Discourse, (chap. 3), Routlegde, pp. 37-51.

Lury C., (2004), Brand : The Logos of the Global Economy, London, Routledge, 198 p.

Lutz F. W., (1982), Tightening up Loose Coupling in Organizations of Higher Education, in Administrative Science Quarterly, vol. 27, $\mathrm{n}^{\circ}$ 4, pp. 653-669.

Moor L., (2007), The rise of brands, Oxford, Berg, $192 \mathrm{p}$.

Moor L. et Lury C., (2010), Brand valuation and topological culture, in M. Aronczyk et D. Powers (éds.), Blowing up the brand, New York, Peter Lang., pp. 29-53.

Nicolini D., (2009), Zooming in and out : Studying practices by switching theoretical lenses and trailing connection, in Organization Studies, 30(12), pp. 1391-1418.

Papilloud C., (2007), La société collaborative : Technologies digitales et lien social, Paris, L'Harmattan, $221 \mathrm{p}$.

Pickering A., (1995), The mangle of practice, Chicago, IL, University of Chicago Press, $296 \mathrm{p}$.

Putnam L. L. et Nicotera A. M. (éds.), (2009), Building Theories of Organizations : The Constitutive Role of Communication, Mahwah, NJ, Erlbaum/Routledge, 240 p.

Schwartzman H. B., (1993), Ethnography in organizations, Qualitative Research Methods Series n 27, Newbury Park, SAGE Publication.

Searle J. R., (1995), The Construction of Social Reality, New York, Free Press, 256 p. 
Stensaker B., (2007), The relationship between branding and organisational change, in Higher Education Management and Policy, 19(1), pp. 13-29.

Tarde G., (2000, [1899]), Social Laws, Kitchener : Batoche Books, 213 p.

Taylor J. R., Cooren F., Giroux N. et Robichaud D., (1996), The Communicational Basis of Organization: Between the Conversation and the Text, in Communication Theory, 6, pp. 1-39.

Wæraas A. et Solbakk M. N., (2008), Defining the essence of a university : lessons from higher education branding, in Higher Education, 57(4), pp. 449-462.

Weick K., (1976), Educational organizations as loosely coupled systems, in Administrative Science Quarterly, 21, pp.1-19.

Weick K. E. et Quinn R. E., (1999), Organizational change, in Annual Review of Psychology, nº 50.

\section{NOTES}

1. L'usage du préfixe re, permet d'insister sur l'effort nécessaire pour que la marque traverse le temps en passant d'un instant $t$ à $t+1$ (Latour, sous presse).

\section{RÉSUMÉS}

La marque est étudiée ici comme un mode d'organisation des représentations à travers une approche verticale-ascendante consistant à expliquer l'ordonnancement social à partir d'interactions. Dans cette perspective, le branding est conçu comme un processus interactionnel des pratiques spécifiques de production, de consommation et de distribution d'éléments de marque à travers lesquels les acteurs représentent l'organisation. À partir de l'étude de la production d'un site web dans un contexte universitaire, nous montrons comment ces pratiques soulèvent des questions d'identités individuelle et collective. De cette manière notre étude se positionne au-delà des habituelles problématiques commerciale, économique ou encore juridique du marketing.

Taking a bottom-up approach, which consists of studying social ordering starting from interactions, this paper presents a study of the brand as a mode of organizing representation. Following this approach, we define branding as an interactional process consisting of specific practices of production, consumption, and distribution of the brand through which actors represent the organization. Based on the study of website production in a university context, we show how these practices confront actors with individual and collective identity issues. In doing so, it goes beyond the usual commercial, economic, and legal problematizations found in marketing studies. 
INDEX

Keywords : branding, communicative constitution of organization, approach organizational identity/image, practice, process

Mots-clés : branding, communication constitutive des organisations, identité et image organisationnelles, pratique, processus

\section{AUTEURS}

\section{BENOIT CORDELIER}

Chaire de relations publiques et communication marketing - Université du Québec à Montréal Benoit Cordelier est professeur au Département de communication sociale et publique de l'université du Québec à Montréal (UQAM), chercheur à la Chaire de relations publiques et communication marketing et au Centre de recherche sur la communication et la santé (ComSanté) ainsi que chercheur associé au MICA (EA 4426, axe Communication, organisations et société, université de Bordeaux), directeur de la Revue internationale de communication sociale et publique (RiCSP) de l'Université du Québec à Montréal (UQAM), Canada. Ses recherches portent sur les logiques de transaction en innovation organisationnelle, le lien social et la relation marchande dans les communautés en ligne, la pragmatique de la publicité et le branding. Adresse électronique : cordelier.benoit@uqam.ca.

\section{VIVIANE SERGI}

Chaire de relations publiques et communication marketing - Université du Québec à Montréal Viviane Sergi est professeure en management à l'ESG UQAM. Ses recherches portent sur les modes d'organisation temporaires, la place et le rôle des textes dans la vie organisationnelle ainsi qu'au leadership. Elle est chercheure associée au Advanced studies of projects and project-based organizations research group (KTH, Stockholm), membre du Groupe d'étude sur la pratique de la stratégie (GéPS) de HEC Montréal et membre de la Chaire en relations publiques et communication marketing de l'UQAM. Adresse électronique : sergi.viviane@uqam.ca. 